\title{
Pruritus as a Presenting Symptom of FIPILI-PDGFRA-Positive Chronic Eosinophilic Leukemia
}

\author{
Mohammad Abu-Tineh (I) \\ Esra'a Aljaloudi ${ }^{2}$ \\ Mohamed A Yassin (iD) \\ 'Department of Oncology- Hematology \\ and BMT Section, National Center for \\ Cancer Care and Research, Hamad \\ Medical Corporation, Doha, Qatar; \\ ${ }^{2}$ Department of Family Medicine, Hamad \\ Medical Corporation, Doha, Qatar
}

\begin{abstract}
Eosinophilia can be found in a variety of benign and malignant conditions, and a persistent eosinophilic count of more than $1500 / \mathrm{mm}^{3}$ necessitates additional investigation. Patients with FIP1L1-PDGFRA-positive chronic eosinophilic leukemia might present as asymptomatic or in a catastrophic state with multi-organ involvement. We present the case of a young male patient who was diagnosed with FIP1L1-PDGFRA chronic eosinophilic leukemia after a long history of recurrent cutaneous symptoms with no systemic signs.
\end{abstract}

Keywords: chronic eosinophilic leukemia, FIP1L1-PDGFRA, eosinophilia, imatinib

\section{Introduction}

FIP1L1-PDGFRA-positive chronic eosinophilic leukemia is a rare myeloproliferative neoplasm marked by a continuous increase in circulating eosinophils ( $\geq 1.5 \times 109 / \mathrm{L})$. Secondary causes should be ruled out before verifying clonality and confirming the CEL diagnosis. ${ }^{1}$ The infiltration of eosinophils into many organs, as well as the production of eosinophilic granules and cytokines, cause severe damage and probable organ malfunction. Patients can report anything from serious symptoms like restricted cardiomyopathy to long-term eosinophilia with no obvious symptoms. According to patients with FIP1L1-PDGFRA-positive chronic eosinophilic leukemia, the gastrointestinal system, lungs, and skin are the most affected organs. ${ }^{1}$ We present the case of a 30-year-old male patient who was diagnosed with FIP1L1-PDGFRA-positive CEL, which manifested mostly as severe pruritus of the skin.

\section{Case Presentation}

A 29-year-old male patient with a background of newly developed attacks of cough and shortness of breath of a few months' duration was diagnosed with intermittent asthma, with no similar history during childhood. The patient was referred to the Dermatology team with complaints of multiple skin lesions related to sun exposure along with persistent pruritus. The patient had an initial assessment of common causes of allergies which came back negative, thus he was referred for further evaluation. A few months later the patient presented with the same complaints, but the later episode was associated with watery diarrhea and a history of subjective weight loss, along with a history of severe persistent pruritus. CBC showed elevated WBC counts with marked eosinophilia for which the patient was referred for further workup. $\mathrm{CBC}$ showed WBC of $22 \times 10^{\wedge} 3 / \mu \mathrm{L}$ normal value

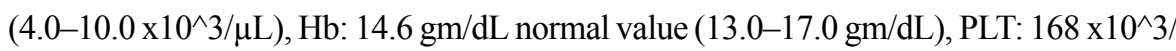

Correspondence: Mohammad Abu-Tineh Department of Oncology- Hematology and BMT Section- National Center for Cancer Care and Research, Hamad Medical Corporation, Alrayan Street, Doha, Qatar

Tel +97455003969

Email Abutineh90@gmail.com 
$\mu \mathrm{L}$ normal value $\left(150-400 \times 10^{\wedge} 3 / \mu \mathrm{L}\right)$, ANC: $4.8 \times 10^{\wedge} 3 / \mu \mathrm{L}$ normal values $\left(2.0-7.0 \times 10^{\wedge} 3 / \mu \mathrm{L}\right)$, eosinophil count: 14.1 $\mathrm{x} 10^{\wedge} 3 / \mu \mathrm{L}$ normal values $\left(0.0-0.5 \times 10^{\wedge} 3 / \mu \mathrm{L}\right)$. Peripheral smear

showed normocytic normochromic red cells, leukocytosis with marked eosinophilia, composed mostly of mature forms, the majority of eosinophils showed abnormal nuclear segmentation and or sparse cytoplasmic granulation, platelets were adequate.

Chest X-ray was unremarkable, abdomen US was significant for mild hepatosplenomegaly.

$\operatorname{IgE}$ levels were within normal limits. Bone marrow was remarkably hypercellular (almost $100 \%$ cellularity) with granulocytic hyperplasia and remarkably increased eosinophilic cells with adequate erythropoiesis and megakaryocytes with some dysplastic forms. CD117 immunostain performed on bone marrow showed increased mast cells, including some spindle-forms. These mast cells were positive for mast cell tryptase and aberrantly positive for CD 25 . No increase in CD34-positive cells was noted. Reticulin stain showed areas of mildly increased reticulin fibers (MF0-1). FISH (fluorescence in situ hybridization) analysis using the FIP1L1/CHIC2/ PDGFRA (4q12) probe was consistent with a deletion of CHIC2 resulting in the fusion of PDGFA and FIP1L1 in 76\% of nuclei. There was no BCR/ABL1 gene rearrangement. Molecular genetics revealed no KIT mutation. The molecular studies for JAK-2, CALR, and KIT D816V mutation were negative.

The patient was started on imatinib $100 \mathrm{mg}$ orally once a day, and his numbers were normalized within a month, along with a considerable improvement in his skin manifestations in terms of frequency, episodes, and intensity.

\section{Discussion}

Eosinophilia is described as an increase in the number of eosinophils in the peripheral blood, with an absolute count of greater than 500 eosinophils $\times 103 \mu \mathrm{L}$. Hypereosinophilic syndrome is defined as an elevated eosinophil count (>1500 eosinophils x103/L) that lasts longer than 6 months (HES). Nonetheless, in 2011, the working conference on eosinophil disorders and syndromes proposed that at least two episodes of eosinophilia separated by at least four weeks can be classified as persistent, indicating that there is no identifiable etiology for eosinophilia and that patients had signs and symptoms of organ involvement. ${ }^{1,2}$

Chronic unexplained eosinophilia greater than 1500/ mm3 is a hallmark of PDGFRA-associated chronic eosinophilic leukemia, a type of blood cancer. Persistent eosinophilia $(1.5 \times 109 / \mathrm{L})$ in the absence of reactive causes, followed by a positive peripheral blood screening for the FIP1L1-PDGFRA gene fusion (via RT-PCR or interphase/ metaphase FISH), is classified as myeloid/lymphoid neoplasms associated with eosinophilia and PDGFRA rearrangement, according to the WHO 2017 classification. The real incidence of chronic eosinophilic leukemia, not otherwise described, is unknown due to the difficulties in separating it from the idiopathic hypereosinophilic syndrome. ${ }^{1}$

CEL is classified as a myeloproliferative subtype of hypereosinophilic syndrome. Eosinophilic myeloid and lymphoid neoplasms with PDGFRA, PDGFRB, and FGFR1 rearrangements were classified as a distinct class in the 2008 WHO classification; ${ }^{3}$ these neoplasms are defined by overexpression of an abnormal tyrosine kinase caused by a mutation or a particular fusion gene. A mutant pluripotent (myeloid-lymphoid) stem cell has been identified as the cell of origin. ${ }^{4}$ Clonal eosinophilia is caused by gene rearrangements or point mutations in the PDGFRA, PDGFRB, or FGFR1 genes, with the creation of a FIP1L1-PDGFRA fusion gene being the most common, ${ }^{5}$ accounting for 10 $20 \%$ of patients with unexplained eosinophilia in Western countries. As a result of the interstitial deletion of $4 \mathrm{q} 12$ that leads to FIP1L1-PDGFRA fusion, approximately $70 \%$ of patients with PDGFRA rearrangement have eosinophilia. ${ }^{6}$

The FIP1L1-PDGFRA fusion protein is a plateletderived growth factor receptor that remains active even when platelet-derived growth factor is present because the first 29 amino acids of the FIP1L1 protein can activate the PDGFR $\alpha$ kinase domain. The activation of the entire subsequent signaling pathway is triggered by the continual phosphorylation of the receptor on a tyrosine, causing the change of hematopoietic cells to an endless growth state. ${ }^{7}$

The FIP1L1-PDGFRA fusion gene is considered the most important molecular biomarker, and it has recently been shown to be susceptible to tyrosine-kinase inhibitor medications like imatinib.

The clinical manifestation is caused by a rapid increase in eosinophils and their distribution in organs such as the skin. Cough, dyspnea, generalized weakness, skin rash, and rhinitis are the most common symptoms. ${ }^{8}$ Persistent eosinophilia can affect any organ; one well-known example is cardiac involvement, notably endomyocardial fibrosis, which increases the risk of death. Lung fibrosis, thromboembolism, and eosinophilic gastritis are some of the most serious symptoms. ${ }^{8}$ In addition to an enlarged spleen and higher tryptase levels in the blood, those with PDGFRA-associated chronic eosinophilic leukemia may have an enlarged spleen. 
Our patient's symptoms were mostly cutaneous, with several skin lesions reported as recurrent skin abscesses connected to intermittent sun exposure, as well as recurrent pruritus that became prominent and severe at the time of diagnosis. There were no major systemic manifestations. Eczema-like symptoms, angioedema, and numerous mucosal ulcers are all common cutaneous signs of eosinophilic leukemia. ${ }^{9}$ Currently, many studies have demonstrated a significant outcome with complete hematologic and molecular remission in FIP1L1PDGFRA-positive patients after initiating imatinib therapy of 100 to $400 \mathrm{mg}$ daily. $^{2}$ A maintenance dose of 100 $200 \mathrm{mg}$ weekly can maintain total metabolic remission, with the majority of patients achieving complete molecular remission with $100 \mathrm{mg}$ daily. ${ }^{10}$

\section{Conclusion}

FIP1L1-PDGFRA-positive CEL can manifest primarily as a skin symptom rather than a systemic disease; this case was reported to raise physician awareness of a common skin symptom that could be a sign of a serious illness; unusual presentations of skin symptoms that do not improve with standard treatment methods should prompt further evaluation.

\section{Abbreviations}

PDGFRA, platelet-derived growth factor receptor A; PDGFRB, platelet-derived growth factor receptor Beta; FGFR1, fibroblast growth factor receptor 1; TKI, tyrosine kinase inhibitor; DM, diabetes mellitus; ANC, absolute neutrophil count; FISH, fluorescence in situ hybridization; HES, hypereosinophilic syndrome; CML, Chronic Myelogenous Leukemia; CBC, complete blood count; MPN, myeloproliferative neoplasm.

\section{Statement of Ethics}

The case was approved by Hamad Medical Corporation Research Center with reference number MRC-04-20-997.

\section{Consent}

Written informed consent was obtained from our patient to allow the publication of information.

\section{Acknowledgment}

We thank Qatar National Library for funding this article.

\section{Disclosure}

The authors report no conflict of interest in this work.

\section{References}

1. Gotlib J. World Health Organization-defined eosinophilic disorders: 2017 update on diagnosis, risk stratification, and management. $\mathrm{Am}$ J Hematol. 2017;92(11):1243-1259. doi:10.1002/ajh.24880

2. Abu-Tineh M, Amer A, Ibrahim F, Ibrahim WH, Alhasson H, Yassin MA. FIP1L1-platelet-derived growth factor receptor A-positive chronic eosinophilic leukemia showing dramatic response to imatinib: a case report. Medicine. 2021;2(1):e0019.

3. Bain BJ. Myeloid and lymphoid neoplasms with eosinophilia and abnormalities of PDGFRA, PDGFRB or FGFR1. Haematologica. 2010;95(5):696. doi:10.3324/haematol.2009.021675

4. Wang SA. The diagnostic work-up of hypereosinophilia Pathobiology. 2019;86(1):39-52. doi:10.1159/000489341

5. Savage N, George TI, Gotlib J. Myeloid neoplasms associated with eosinophilia and rearrangement of PDGFRA, PDGFRB, and FGFR 1: a review. Int J Lab Hematol. 2013;35(5):491-500. doi:10.1111/ijlh.12057

6. Vandenberghe P, Wlodarska I, Michaux L, et al. Clinical and molecular features of FIP1L1-PDFGRA (+) chronic eosinophilic leukemias. Leukemia. 2004;18(4):734-742. doi:10.1038/sj.leu.2403313

7. Gotlib J, Cools J, Malone JM, Schrier SL, Gilliland DG, Coutré SE. The FIP1L1-PDGFR $\alpha$ fusion tyrosine kinase in hypereosinophilic syndrome and chronic eosinophilic leukemia: implications for diagnosis, classification, and management. Blood. 2004;103 (8):2879-2891. doi:10.1182/blood-2003-06-1824

8. Roufosse F, Weller PF. Practical approach to the patient with hypereosinophilia. J Allergy Clin Immunol. 2010;126(1):39-44. doi:10.1016/j.jaci.2010.04.011

9. Leiferman KM, Gleich GJ, Peters MS. Dermatologic manifestations of the hypereosinophilic syndromes. Immunol Allergy Clin North Am. 2007;27(3):415-441. doi:10.1016/j.iac.2007.07.009

10. Reiter A, Gotlib J. Myeloid neoplasms with eosinophilia. Blood. 2017;129(6):704-714. doi:10.1182/blood-2016-10-695973

\section{Publish your work in this journal}

The Journal of Blood Medicine is an international, peer-reviewed, open access, online journal publishing laboratory, experimental and clinical aspects of all aspect pertaining to blood based medicine including but not limited to: Transfusion Medicine; Blood collection, Donor issues, Transmittable diseases, and Blood banking logistics; Immunohematology; Artificial and alternative blood based therapeutics; Hematology; Biotechnology/nanotechnology of blood related medicine; Legal aspects of blood medicine; Historical perspectives. The manuscript management system is completely online and includes a very quick and fair peer-review system. Visit http://www.dovepress.com/testimonials.php to read real quotes from published authors. 